\title{
Neural Representations of Individual Stimuli in Humans Revealed by Gamma-Band Electrocorticographic Activity
}

\author{
Joshua Jacobs and Michael J. Kahana \\ Department of Psychology, University of Pennsylvania, Philadelphia, Pennsylvania 19104
}

A fundamental question in neuroscience concerns how the human brain represents perceptual and conceptual information. Traditionally, researchers probed this issue by identifying single neurons that increased their firing rate when an animal encountered certain stimuli. Here we provide evidence of a complementary scheme in which gamma-band (25-128 Hz) electrocorticographic (ECoG) activitya phenomenon involving large groups of neurons- encodes the active cognitive representation. We analyzed intracranial brain recordings from neurosurgical patients while they studied lists of visually presented letters and found that the amplitude of gamma-band activity encoded the identity of the current letter. These letter-specific patterns occurred during periods of overall increased gamma amplitude and were linked to the phase of simultaneous theta (4-8 Hz) oscillations. In occipital cortex, these patterns sometimes reflected the shape of the viewed letter, but, in other brain regions, this phenomenon was not related to letter form. Our findings show that gamma-band activity encodes a range of perceptual and conceptual information, suggesting that ECoG recordings can reveal neural correlates of specific human cognitive representations.

\section{Introduction}

The question of how the brain represents percepts and memories has long occupied center stage in philosophy, psychology, and neuroscience (Galton, 1883; Lashley, 1950; Melton and Martin, 1972). A prominent hypothesis arising from both human and animal studies is that the brain represents behavior-related information via synchronized patterns of activity across large groups of neurons (Hebb, 1949). One way of probing these large-scale patterns is the electrocorticogram (ECoG), which measures synaptic and spiking activity across neuronal populations with a high temporal resolution (Logothetis et al., 2001). In particular, ECoG activity in the gamma frequency band $(25-128 \mathrm{~Hz})$ identified brain regions that are involved in various perceptual, motor, and cognitive processes. For example, after tactile stimulation, the amplitude of gamma activity transiently increases in somatosensory regions (Bauer et al., 2006; Ray et al., 2007), and, during movement, gamma activity increases in motor cortex (Crone et al., 1998; Miller et al., 2007). Beyond sensorimotor processing, gamma ECoG activity has also identified the brain regions involved in language processing (Canolty et al., 2007) and in episodic (Sederberg et al., 2007) and working (van Vugt et al., 2009) memory.

In addition to probing overall differences between brain structures, a different set of studies examined detailed recordings

\footnotetext{
Received May 8, 2009; revised June 18, 2009; accepted July 8, 2009.

This work was sponsored by National Institutes of Health Research Grants MH61975, MH062196, and NS50067 and National Science Foundation Grant SBE0354378. We are thankful to Marieke van Vugt, Christoph Weidemann, and Per Sederberg for extensive technical assistance, Sean Polyn, Lyle Ungar, and Jeremy Manning for thoughtful discussions, and Drs. Brian Litt, Gordon Baltuch, Andreas Schulze-Bonhage, and Joe Madsen for providing us with data.

Correspondence should be addressed to Dr. Joshua Jacobs, University of Pennsylvania, 3401 Walnut Street, Room 303C, Philadelphia, PA 19104. E-mail: jojacobs@psych.upenn.edu.

DOl:10.1523/JNEUROSCI.2187-09.2009

Copyright $\odot 2009$ Society for Neuroscience $\quad$ 0270-6474/09/2910203-12\$15.00/0
}

of gamma activity within particular regions, identifying gamma patterns that are correlates of specific neuronal-network states (Freeman, 1991; Singer and Gray, 1995). For example, in visual cortex, patterns of gamma phase synchrony across distinct cortical columns distinguished visual scenes with different global arrangements (Eckhorn et al., 1988; Gray et al., 1989). Furthermore, in auditory cortex, intercolumn differences in gamma amplitude encoded the learned category of a percept (Ohl et al., 2001).

Together, these studies showed that gamma-band activity both identifies active brain regions and reveals detailed properties of the current state of a neuronal network. Motivated by this, we examined whether gamma-band activity in humans revealed neural patterns that underlie the cognitive representations of individual stimuli. This is an important step in testing the claim that gamma-band activity underlies a wide range of cognitive processes, possibly including conscious awareness (Singer and Gray, 1995; Llinás et al., 1998; Tononi and Koch, 2008).

We examined ECoG recordings from neurosurgical patients studying lists of letters in a working-memory task. Our primary interest was examining neuronal activity that distinguished the identity of the currently viewed letter. After each letter presentation, at many electrodes, we found that the amplitude of gammaband ECoG activity increased. During this increase, the precise amplitude of gamma activity often distinguished the identity of the currently viewed letter. These letter-specific gamma patterns appeared in occipital and temporal brain regions and were linked to the phase of simultaneous theta $(4-8 \mathrm{~Hz})$ oscillations. These findings are not necessarily indications of brain regions that are exclusively devoted to letter processing; rather, they suggest that gamma activity is a general phenomenon that reveals stimulus-specific neuronal processes throughout various cortical regions. 


\section{Materials and Methods}

Patients. We analyzed data from 37 patients undergoing invasive monitoring as treatment for drug-resistant epilepsy. (Data from four additional patients were excluded as a result of technical issues.) During their 1-3 week invasive monitoring periods, these patients volunteered to participate in our cognitive testing in their free time between clinical procedures. Each patient participated in between one and five testing sessions. Our research protocol was approved by the appropriate institutional review boards at the Hospital at the University of Pennsylvania (Philadelphia, PA), Children's Hospital (Philadelphia, PA), University Clinic (Freiburg, Germany), Children's Hospital (Boston, MA), and Brigham and Women's Hospital (Boston, MA). Informed consent was obtained from patients (or their guardians, in the case of children). Some of these datasets have been examined previously (Raghavachari et al., 2001; Howard et al., 2003; Rizzuto et al., 2003; van Vugt et al., 2009), but the data analyses described here are novel.

Electrophysiology. In each patient, we analyzed ECoG recordings from 19 to 126 electrodes. Electrodes were positioned by clinical teams to identify seizure foci and functional brain regions to guide potential subsequent resective surgery. As a result, a large proportion of electrodes were typically placed in temporal cortex, but many electrodes were also placed in the hippocampi and frontal, occipital, and parietal cortices (for details, see supplemental table, available at www.jneurosci.org as supplemental material). Because the clinical procedure of identifying seizure foci involves placing electrodes in any region that is merely potentially epileptogenic, the majority of recordings come from brain regions outside the area that is subsequently determined to be involved in seizures (Sederberg et al., 2003; Jacobs et al., 2009). Recording electrodes typically consisted of two configurations: grid and strip electrodes, which are placed on the surface of the neocortex, and depth electrodes, which penetrate through the cortex and record from hippocampus and overlying regions. Electrode locations were computed by coregistering a postoperative computed tomography scan with a higher-resolution preoperative magnetic resonance image. These images were then normalized to a standard-sized brain, and the lobe and Brodmann area of each electrode were computed using Talairach coordinates (Talairach and Tournoux, 1988; Lancaster et al., 2000). We used brain images from the WFU PickAtlas for data visualization (Maldjian et al., 2003).

ECoG activity was recorded at a sampling rate of $250-1024 \mathrm{~Hz}$ using Bio-Logic, XLTek, Neurofile, or Nicolet recording systems, depending on the testing hospital. These recordings were synchronized with measurements of the patient's behavior in the task via optically isolated synchronization pulses received from the testing computer on a spare recording channel. To eliminate potential electrical noise, when analyzing the gamma band exclusively, recordings were notch filtered using a zero-phase-distortion Butterworth filter at $60 \mathrm{~Hz}$ (United States) or $50 \mathrm{~Hz}$ (Europe). The recording from each electrode was rereferenced to the average of neighboring electrodes from the same grid, strip, or depth probe.

Task. During each $\sim 45$ min testing session, patients participated in a working-memory task (Sternberg, 1966). In each trial of the task, patients were presented with a list of one to six letters on the screen of a bedside laptop computer. During this presentation portion of the trial, first a fixation cross appeared, and then the letters were displayed sequentially on the computer screen. Each letter was on screen for $700 \mathrm{~ms}$, followed by 275-350 ms (uniformly distributed) of blank screen, for a total of a 975-1050 ms interstimulus interval. Patients were instructed to closely attend to each stimulus presentation and to silently hold the identity of each item in memory. The letter lists included only consonants (i.e., no vowels) to prevent patients from using mnemonic strategies (e.g., remembering the entire list as an easily pronounceable word-like sound). After the presentation of each list, the response period began when a probe item was displayed. Then patients responded by pressing a key to indicate whether the probe was present in the just-presented list or whether it was absent. After the key press, the computer indicated whether the response was correct, and then a new list was presented. Across all letter presentations, some patients were presented with all 19 consonants, whereas others only viewed a 16 letter subset. On average, across all sessions and trials, each patient was presented with 720 letters. Because of the limitations of the hospital testing environment, we were unable to measure patients' eye movements during the task. However, we frequently reminded patients to fixate their gaze to minimize unnecessary eye movements.

Data analysis. Our goal was to identify patterns of neuronal activity related to the identity of individual letters. Thus, we exclusively focused on data from the presentation portion of each trial. We excluded the response period because we did not want our results to include the act of comparing the probe with the contents of memory. First, we resampled all ECoG recordings to $500 \mathrm{~Hz}$, to provide consistency across data from different recording systems. We analyzed ECoG activity in the time period after each letter presentation using two forms of spectral analysis: (1) Morlet wavelets and (2) bandpass filtering in conjunction with the Hilbert transform. We used Morlet wavelets (wave number of 6) to systematically examine oscillatory activity across a wide range of frequencies $\left(2^{x / 8} \mathrm{~Hz}\right.$ for $\left.x \in 8, \ldots, 56\right)$, identifying the bands exhibiting stimulusrelated activity (Fig. 1). Subsequently, we used bandpass filtering and the Hilbert transform to directly analyze oscillatory patterns across these relatively broad frequency bands (Freeman, 2007). This is important when analyzing nonstationary ECoG patterns, such as gamma oscillations, which often appear throughout wide frequency ranges (Bressler and Freeman, 1980; Siegel and König, 2003). We used this scheme to analyze two distinct ranges: the $65-128 \mathrm{~Hz}$ high-gamma band and the $25-55 \mathrm{~Hz}$ low-gamma band (Fig. $1 D$ ). To perform this procedure, first we filtered the raw ECoG signal in each of these ranges using a secondorder Butterworth bandpass filter. Next, we applied the Hilbert transform, which yields a complex number, and took the absolute value to extract the instantaneous amplitude of the signal (see Fig. $3 B, D$, black lines). We smoothed the gamma-amplitude time series of each trial with a $100 \mathrm{~ms}$ boxcar filter to compensate for trial-to-trial jitter.

To identify oscillatory activity that distinguished the viewing of different letters, we used a statistical framework based on a one-way ANOVA. This ANOVA tested the null hypothesis that the oscillatory amplitude did not vary according to the identity of the viewed letter. We labeled each trial with the identity of the currently viewed consonant; these labels formed the groups for the ANOVA. Then, at each time point, we performed an ANOVA to compute the probability of observing these data under the null hypothesis that the amplitude of gamma-band oscillatory activity did not differ between these groups. These ANOVAs were performed separately for every electrode in both the high- and low-gamma frequency bands.

We used a nonparametric clustering-based procedure to estimate the statistical significance of the ANOVA (Maris and Oostenveld, 2007). This procedure identifies contiguous time windows of letter-related gamma activity and accounts for the fact that we are repeatedly applying ANOVAs at multiple time points. Critically, this procedure allows us to avoid a priori assumptions about particular time points that might exhibit letter-specific activity. Analyzing multiple ANOVA $p$ values over time is problematic because each ANOVA is conducted independently, but the underlying neural data are correlated in time. This clustering procedure solves this problem by identifying contiguous epochs of significant letter-related activity and assessing statistical significance via a permutation test. This is a powerful and appropriate technique for this dataset, because we expected that any letter-specific oscillatory activity would appear for multiple consecutive time points.

To conduct this procedure, we first performed a separate one-way ANOVA (factor was identity of viewed letter) at each time point, and we then identified contiguous time windows (clusters) in which the ANOVA $p$ values were $<0.01$. Next, for each cluster, we computed a cluster statistic, by taking the sum (over time) of the absolute values of all the inverse-normal-transformed $p$ values ( $Z$ scores) in that cluster. Electrodes that exhibited robust letter-specific activity were identified as those having at least one cluster with a very large cluster statistic. Large cluster statistics arose in two ways: highly significant letter-specific activity (very small ANOVA $p$ values) or weaker letter-specific activity that persisted for a long duration. The statistical significance of any observed clusters was assessed by performing the same procedure on a shuffled version of this dataset in which the viewed letter in each trial was ran- 
A
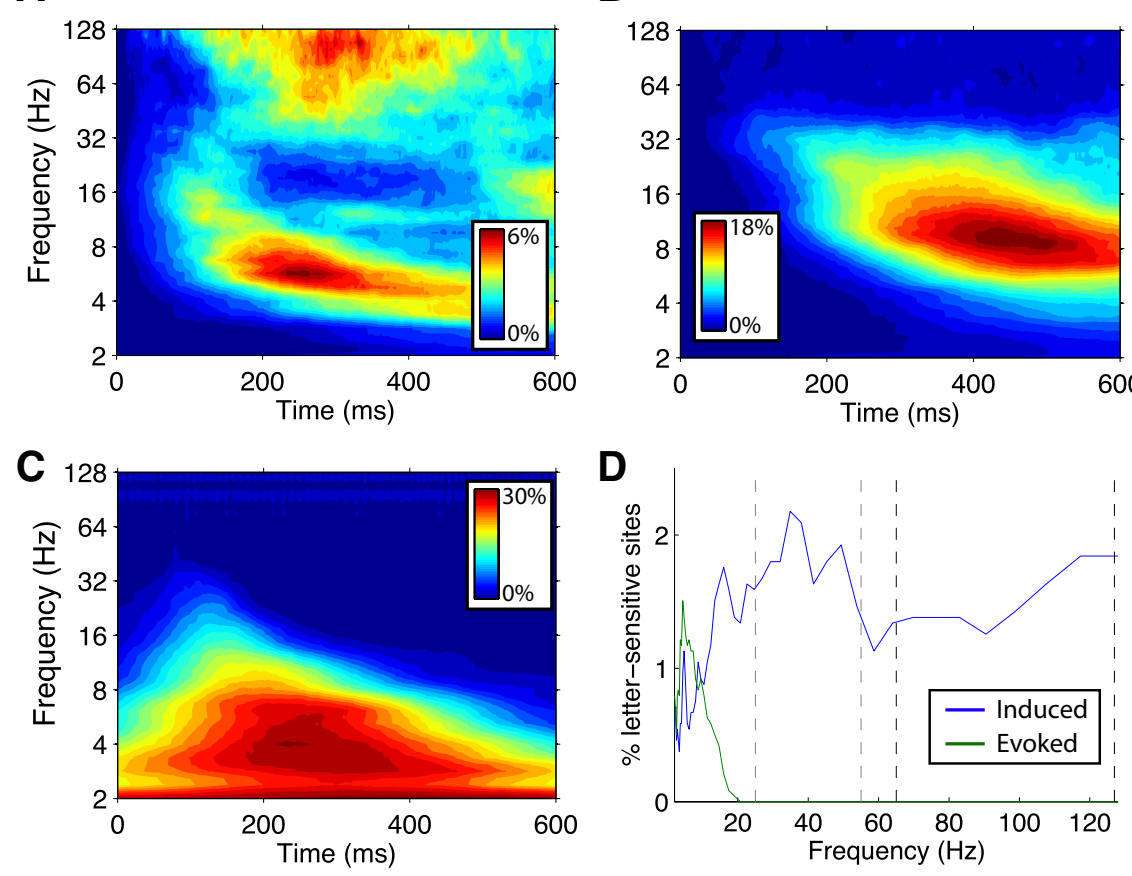

Figure 1. Oscillatory ECOG activity after stimulus presentation. $\boldsymbol{A}$, Percentage of electrodes exhibiting a significant increase in oscillatory power after stimulus presentation ( $p<0.01$, rank-sum test), at each poststimulus time point and frequency. $B$, Percentage of electrodes exhibiting a significant decrease in oscillatory power after stimulus presentation. $\boldsymbol{C}$, Percentage of electrodes exhibiting significant phase synchrony (Rayleigh test, $p<10^{-3}$ ) at each frequency and time point after stimulus presentation. $\boldsymbol{D}$, Percentage of electrodes with oscillatory power that significantly distinguished the viewed letter (ANOVA, $p<$ 0.01 , based on mean power in each trial at $100-400 \mathrm{~ms}$ after stimulus onset). Blue line indicates non-phase-locked ("induced") activity, and green line indicates phase-locked ("evoked") activity. Black dashed lines depict the 65-128 Hz high-gamma frequency band; gray dashed lines indicate the $25-55 \mathrm{~Hz}$ low-gamma band.

domly determined. In each iteration of the shuffling procedure $(n=$ 1000), the statistic of the largest observed cluster (if any) was saved, thus estimating the distribution of maximum cluster statistics arising by chance at each electrode. The statistical significance of the clusters from the true dataset was determined by comparing the observed cluster statistic from the actual dataset with the 99th percentile of the distribution of maximum cluster statistics from the permuted datasets. If the actual cluster statistic exceeded this threshold, then the cluster was considered significant and the electrode was designated as exhibiting significant letter-specific oscillatory activity. Importantly, this clustering procedure set the false-positive rate at a fixed level per electrode rather than varying with the number of ANOVAs performed over time. If a single electrode exhibited significant letter-specific activity in both the high- and lowgamma bands, we analyzed activity in the band in which the effect was more statistically robust.

We used this permutation procedure to identify brain regions in which the count of electrodes exhibiting letter-specific activity significantly exceeded the number expected by chance. Using the shuffled datasets, we estimated the distribution of the total number of significant electrodes that would be observed by chance in each region. Critically, because we used identical shuffle sequences for all electrodes and frequencies in each patient, this procedure accounted for potential correlations between electrodes and frequency bands. (Applying this procedure in each of five brain regions, we found that $\sim 1.99 \%$ of electrodes exhibited this effect by chance.) We designated a region as exhibiting a significant level of letterspecific activity if the true number of electrodes exhibiting letter-specific effects exceeded the 95th percentile of the distribution expected by chance. The false discovery rate procedure was used to correct for multiple comparisons (Genovese et al., 2002).

For each electrode exhibiting letter-specific activity, we labeled the onset time of letter-specific activity as the earliest time in the first cluster in which the ANOVA $p$ value was $<0.01$ (uncorrected). We labeled the time point of maximal letter-specific activity as the time point within a significant cluster in which the ANOVA $p$ value was smallest. We used post hoc one-sided $t$ tests to identify particular letters that caused a greater level of gamma activity than other letters at the same time point.

We also identified electrodes that exhibited overall gamma-amplitude increases regardless of letter identity. To identify this pattern, we used a rank-sum test to compare the amplitude of oscillatory activity observed at each time point with the amplitude of oscillatory activity at the time of stimulus onset $(t=0)$. We designated any electrodes that exhibited a significant increase $(p<0.001)$ in the amplitude of oscillatory activity for 30 consecutive milliseconds as exhibiting a stimulus-related increase.

We were also interested in assessing the relation between gamma activity and the phase of lower-frequency oscillatory activity (Mormann et al., 2005; Canolty et al., 2006; Tort et al., 2008). To identify oscillatory phase locking to letter presentation, at each time point and frequency, we calculated the phase distribution (across trials) using Morlet wavelets. We used the Rayleigh statistic to test the hypothesis that each phase distribution was uniform. When the $p$ value from this test was $<10^{-3}$, we designated the phase distribution as phase locked (Jacobs et al., 2007). We also analyzed phase locking in the $4-8 \mathrm{~Hz}$ theta band exclusively. To do this, we bandpass filtered the ECoG signal at $4-8 \mathrm{~Hz}$ and then applied the Hilbert transform. Then, at each time point, we calculated the instantaneous theta phase by applying $\tan ^{-1}$ to the output of the Hilbert transform (Freeman, 2007). We computed the Rayleigh test $p$ value of the phase distribution from each time point (across trials) and identified the time point of peak phase locking as the latency associated with the smallest $p$ value.

To examine potential spatial clustering of the electrodes exhibiting letter-specific activity, we used Moran's $I$, a statistic that measures spatial autocorrelation (Moran, 1950). We computed $I$ according to the procedure described by Ripley (1981). A significant positive value of $I$ indicates that observations $X(i)$ and $X(j)$ are positively correlated when electrodes $i$ and $j$ are proximal (as measured by the reciprocal of the Euclidean distance between these electrodes). Electrodes from different patients were considered infinitely distant from each other. We assessed statistical significance using a permutation procedure, which estimated the distribution of $I$ expected by chance using 10,000 random shuffles of the dataset. This shuffling occurred in a manner that permuted electrode locations within each patient, but not across patients, to specifically test for spatial clustering within individuals. When examining spatial correlations in the prevalence of letter-specific activity, we examined all electrodes, setting $X(i)=1$ if electrode $i$ exhibited letter-specific activity and $X(i)=0$ otherwise. When probing spatial correlations in the latency of letter-specific activity, we only included the electrodes that exhibited letter-specific activity, and we set $X(i)$ to the time point when this effect was most significant. When measuring spatial correlations in the frequency in which letter-specific activity appeared, we set $X(i)=1$ for the high-gamma band and $X(i)=0$ for the low-gamma band.

To examine whether the letter-specific gamma activity of an electrode related to visual properties of each letter (i.e., the shape of the letter), we analyzed the mean gamma amplitude for each letter in conjunction with data from a separate psychophysical study of the perceptual discriminability of individual letter pairs (S. T. Mueller and C. T. Weidemann, unpublished observation). This analysis was performed at each electrode that had a significant cluster of letter-related activity, at the time point at 
A

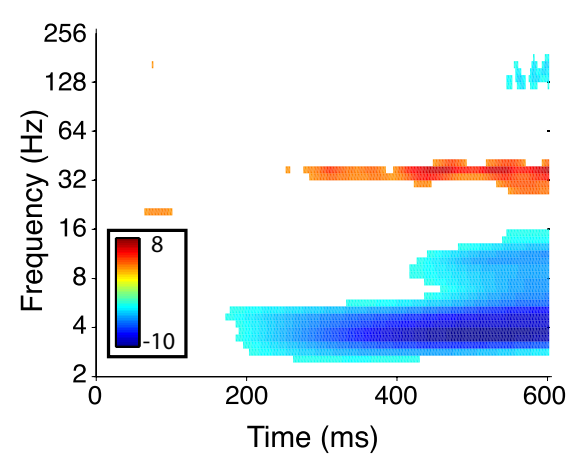

B

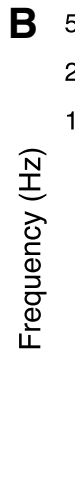

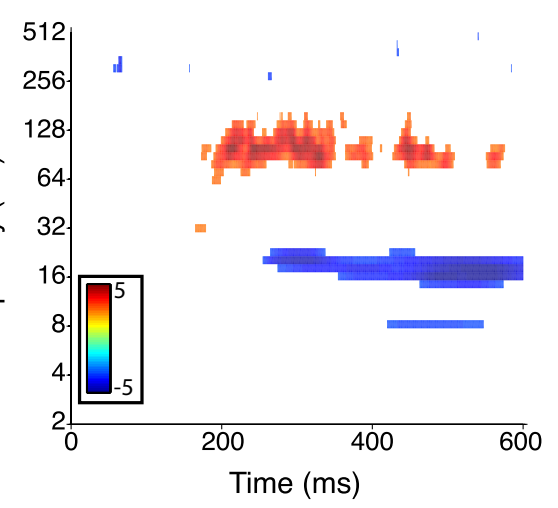

C

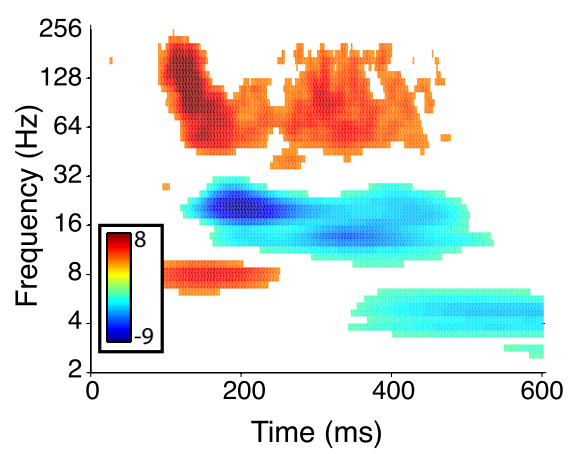

Figure 2. Examples of the oscillatory activity observed at individual electrodes. $A$, The activity of an electrode from patient 18 that showed an increase in low-gamma ( $\sim 40 \mathrm{~Hz}$ ) oscillatory power attributable to stimulus presentation. Coloring indicates the $z$-score (inverse-normal transformation) of the $p$ value (rank-sum test) comparing the power at each time point/frequency with the power at baseline (time $=0$ ). Red coloring indicates significantly increased oscillatory power, and blue indicates significantly decreased oscillatory power ( $p$ values $<0.01$, uncorrected). This plot was created without resampling the original data and thus measured frequencies ranging from 2 to $256 \mathrm{~Hz}$, which is the Nyquist frequency for this patient's $512 \mathrm{~Hz}$ recording. $\boldsymbol{B}$, The activity of an electrode from patient 27 that showed increased high-gamma power attributable to stimulus presentation. This gamma-power increase did not appear beyond $\sim 128 \mathrm{~Hz}$. Measured frequencies range from 2 to $512 \mathrm{~Hz}$, because this recording was sampled at $1024 \mathrm{~Hz}$. C, The activity of an electrode from patient 21 that exhibited increased high-gamma power attributable to stimulus presentation. This power increase extended to nearly $256 \mathrm{~Hz}$. Measured frequencies range from 2 to $256 \mathrm{~Hz}$, because this recording was sampled at $512 \mathrm{~Hz}$.

which this effect was strongest. In the psychophysical study, subjects distinguished which of two letters were displayed on a computer monitor (noise was added to the image of each letter to make discrimination difficult). Discrimination accuracy was computed for each letter pair to indicate the visual similarity between the two letters (high discrimination accuracy indicates that the two letters had very different shapes, and low discrimination accuracy indicates similar-looking letters). Next, to determine whether the amplitude of oscillatory activity related to the shapes of the letters, for each letter pair, we computed the magnitude of the difference between the mean gamma response to each letter. Then we computed the nonparametric correlation (Kendall's $\tau$ ) between the distribution of gamma differences and the distribution of discrimination accuracies (across letter pairs). A significant positive correlation indicates that the electrode's gamma activity related to low-level visual properties of the letter (i.e., similar-looking letters yield similar gamma-band responses.). We assessed statistical significance using 1000 iterations of a permutation procedure.

\section{Results}

We analyzed ECoG recordings from 37 patients as they memorized lists of letters in a working-memory task. Our objective was to identify patterns of brain activity that distinguished the identity of the currently viewed letter. Taking a systematic approach, we first examined the spectrum of ECoG oscillatory activity triggered by letter viewing. For each electrode, we calculated the power spectrum at various time points after letter presentation. We identified electrodes exhibiting significant increases in oscillatory power, at each frequency and time point, relative to the moment before letter appearance (Fig. 1A). This revealed two main patterns: from $\sim 100$ to $\sim 600 \mathrm{~ms}$ after letter onset, there was an increase in the power of gamma-band activity (25-128 $\mathrm{Hz}$ ), and at $\sim 100-400 \mathrm{~ms}$, power increased in the theta band $(4-8 \mathrm{~Hz})$. Many electrodes also exhibited significant stimulusrelated decreases in power in the alpha band $(10-16 \mathrm{~Hz})$ at $\sim 200-600 \mathrm{~ms}$ (Fig. 1B). Because external stimuli can alter the phase of ECoG oscillations (Rizzuto et al., 2003), we examined whether ECoG phase was aligned to letter presentation (Fig. 1C). This revealed different effects for oscillatory activity in the theta and gamma bands, with significant phase locking in the theta band at $\sim 250 \mathrm{~ms}$ for $\sim 30 \%$ of electrodes and no appreciable phase locking in the gamma band.

Next, we examined whether the amplitude of these oscillations encoded information about the identity of the viewed letter.
To the extent that brain oscillations correlate with neuronal spiking (Logothetis et al., 2001), this analysis is analogous to seminal studies in primates that identified neurons responding to specific visual stimuli (Tovée et al., 1993). For each electrode, we computed the mean oscillatory power in each trial, at various frequencies, over the 150-400 ms interval after letter onset. Then, we performed an ANOVA to test whether the amplitude of oscillatory activity, across trials, varied according to the identity of the just-viewed letter. At many electrodes, we could reject the hypothesis that the amplitude of ECoG activity (at a particular frequency) was not related to the identity of the viewed letter (Fig. $1 D$ ). In addition to phase-locked theta-band patterns (Liu et al., 2009), we observed non-phase-locked letter-specific patterns in the gamma band. We focused on these gamma-band patterns because of the extensive experimental and theoretical literatures showing that gamma-band activity is a temporally precise indication of local neuronal activation (Freeman, 1975; Eckhorn et al., 1988; Gray et al., 1989; Logothetis et al., 2001; Miller et al., 2007).

We constructed a computational framework to precisely characterize letter-specific gamma activity (for details, see Materials and Methods). Because gamma oscillations are nonstationary and vary in frequency from one cycle to the next (Bressler and Freeman, 1980; Siegel and König, 2003), we used bandpass filtering to detect gamma oscillations across two broad ranges: the 65-128 Hz high-gamma band and the 25-55 Hz low-gamma band. These two frequency ranges were chosen to capture the diversity of gamma-band activity observed at individual electrodes, including narrow-band oscillations (Fig. 2A,B) and broader spectral patterns, which sometimes appeared at highgamma frequencies (Fig. 2C) (Miller et al., 2007). In each frequency range, we computed the instantaneous amplitude of each filtered signal using the Hilbert transform (Freeman, 2007), and, at each time point, we performed an ANOVA to measure amplitude differences between trials in which different letters were presented. Based on the ANOVA $p$ values, we used a nonparametric clustering statistic (Maris and Oostenveld, 2007) to identify contiguous time periods in which each electrode exhibited letter-related activity. We applied this procedure to all electrodes in our dataset and identified the electrodes with at least one sig- 
A

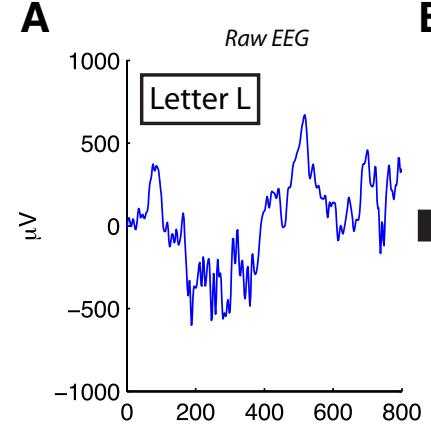

B
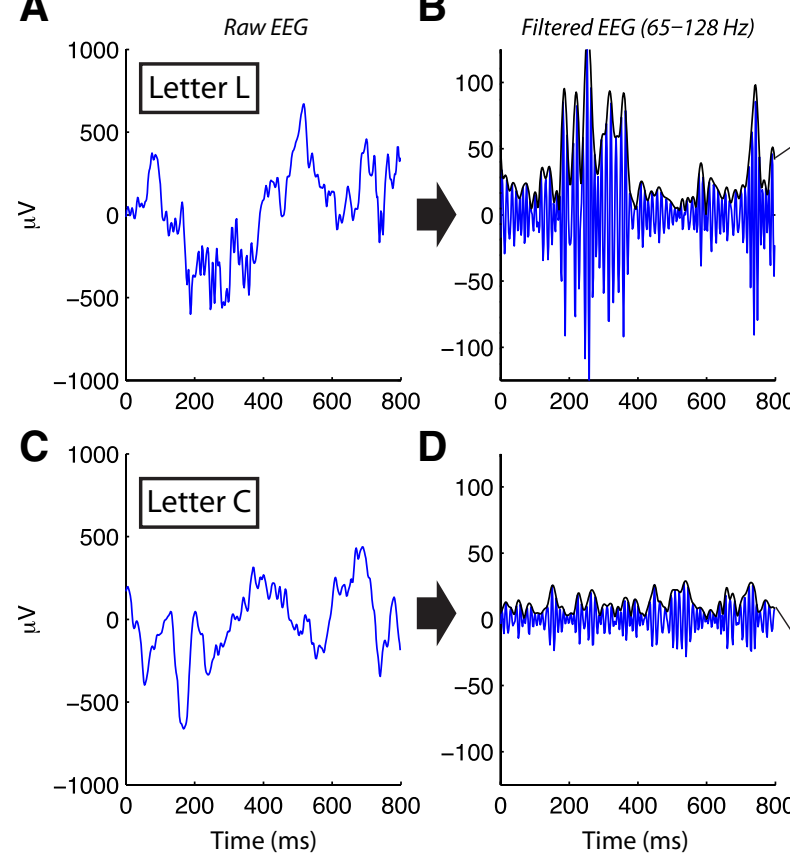

E

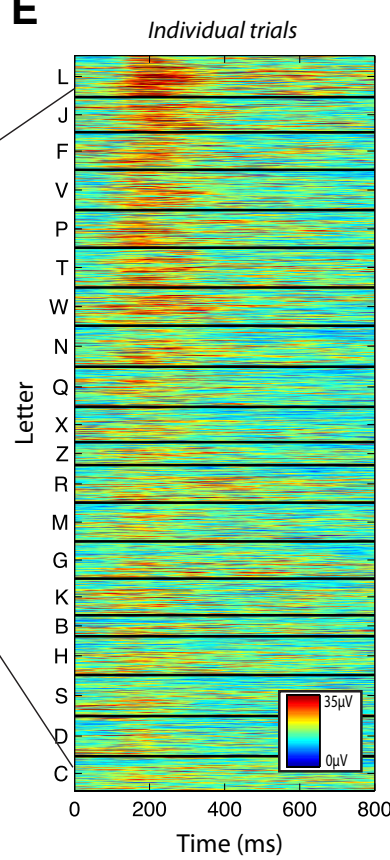

$\mathbf{F}$

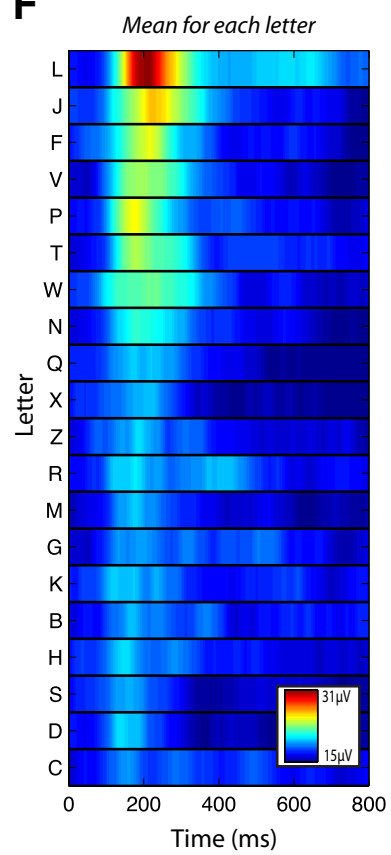

Figure 3. An example of letter-related high-gamma activity. A, A raw ECoG trace at an electrode from patient 20's left temporal cortex (Brodmann area 21) after viewing letter L. B, The EcoG trace from $\boldsymbol{A}$ filtered in the high-gamma band ( $65-128 \mathrm{~Hz}$ ). Black line indicates the instantaneous amplitude of the filtered signal. C, A sample raw ECoG trace after presentation of letter $C$ (same electrode as $\boldsymbol{A}$ ). $\boldsymbol{D}$, The ECoG signal from $\boldsymbol{C}$ filtered in the high-gamma band. $\boldsymbol{E}$, Amplitude of high-gamma ECoG activity at this electrode across all individual trials. Trials are ordered so that presentations of the same letter are contiguous (see labels on vertical axis). $\boldsymbol{F}$, The mean high-gamma EC $0 G$ amplitude for each letter.

nificant cluster of letter-related activity $(p<0.01)$ in either the high- or low-gamma band.

Using this framework, we identified 78 electrodes (of 2330) that exhibited letter-related gamma activity (for details, see supplemental table, available at www.jneurosci.org as supplemental material). As an example, Figure 3 depicts the letter-related highgamma activity observed at an electrode from one patient's left occipital cortex. When this patient viewed the letter $\mathrm{L}$, this electrode exhibited high-amplitude gamma activity (Fig. $3 A, B$ ), whereas after viewing the letter $C$, the amplitude of gamma was lower (Fig. 3C,D). Many letters caused a gamma-amplitude increase at $\sim 200 \mathrm{~ms}$, but the precise magnitude of this increase varied with the identity of the letter (Fig. $3 E, F$ ). The letterspecific activity of this electrode is detailed in Figure $4 A$, which shows that a significant cluster $(p<0.01)$ of letter-specific activity began at a latency of $124 \mathrm{~ms}$ (left panel). The magnitude of this effect was largest at $218 \mathrm{~ms}$, at which the gamma amplitude was significantly elevated for letters L, J, F, V, and P (post hoc $t$ tests, $p<0.05)$

Other electrodes in this patient also exhibited significant letter-related gamma activity. For example, Figure $4 B$ depicts the activity of an electrode from left temporal cortex that exhibited significant letter-related high-gamma activity at similar time points but had maximal activity for different letters ( $\mathrm{T}$ and $\mathrm{W}$ ). Across our dataset, we observed that neighboring electrodes sometimes exhibited letter-related gamma activity at similar time points but that individual sites had maximal activity for different letters. For example, Figure $4 E$ depicts the activity of an electrode that had significantly elevated high-gamma amplitude for letters $\mathrm{F}, \mathrm{Z}$, and $\mathrm{M}$, whereas a neighboring electrode had peak highgamma activity for letters $\mathrm{Q}$ and $\mathrm{L}$ (Fig. $4 F$ ). In particular, the electrode whose activity is depicted in Figure $4 F$ is notable because it had an especially large response for the letter Q. In addition to the letter-specific high-gamma activity described above, we also observed similar patterns in the low-gamma band (Fig. 5). Slightly more of the sites showing letter specificity exhibited this phenomenon in the low-gamma band (60\%) than in the high-gamma band (40\%), although this difference was not statistically reliable (binomial test, $p=0.09$ ).

The examples of letter-specific gamma activity in Figures 4 and 5 showed that letter-specific activity often coincided with an overall increase in gamma-band activity for many letters. Across our entire dataset, we found that letter-specific activity was prevalent at the electrodes that exhibited overall increases in gamma amplitude $\left(\Phi\right.$ correlation coefficient $=0.24 ; \chi_{(1)}^{2}=$ 137; $p<10^{-15}$ ). Among electrodes with overall gammaamplitude increases, 19\% exhibited letter-specific activity, whereas letter-specific activity appeared at approximately chance levels (2\%) at other electrodes. Thus, the phenomenon of letterspecific gamma activity was essentially limited to sites exhibiting overall gamma-amplitude increases.

To understand how overall gamma-amplitude increases related to the emergence of letter-specific activity, we analyzed the temporal relation between these phenomena, at the electrodes exhibiting both patterns. We found that the first occurrence after letter presentation is an overall increase in gamma-band activity, which occurred at a latency of $\sim 200-350 \mathrm{~ms}$. Next, after $56 \pm 24$ ms (mean $\pm \mathrm{SE}$ ), gamma activity begin to significantly encode the identity of the viewed letter (Graboi and Lisman, 2003). The magnitude of these inter-letter differences peaked after another $91 \pm 28 \mathrm{~ms}$.

Recent research has shown that coupling between gamma oscillations and the phase of theta oscillations has an important functional role in neural processing (Chrobak and Buzsáki, 1998; Mormann et al., 2005; Canolty et al., 2006; Tort et al., 2008). Because letter viewing caused theta phase locking (Fig. 1C), we tested whether theta phase related to letter-related gamma-band activity. First, we identified the electrodes that exhibited signifi- 

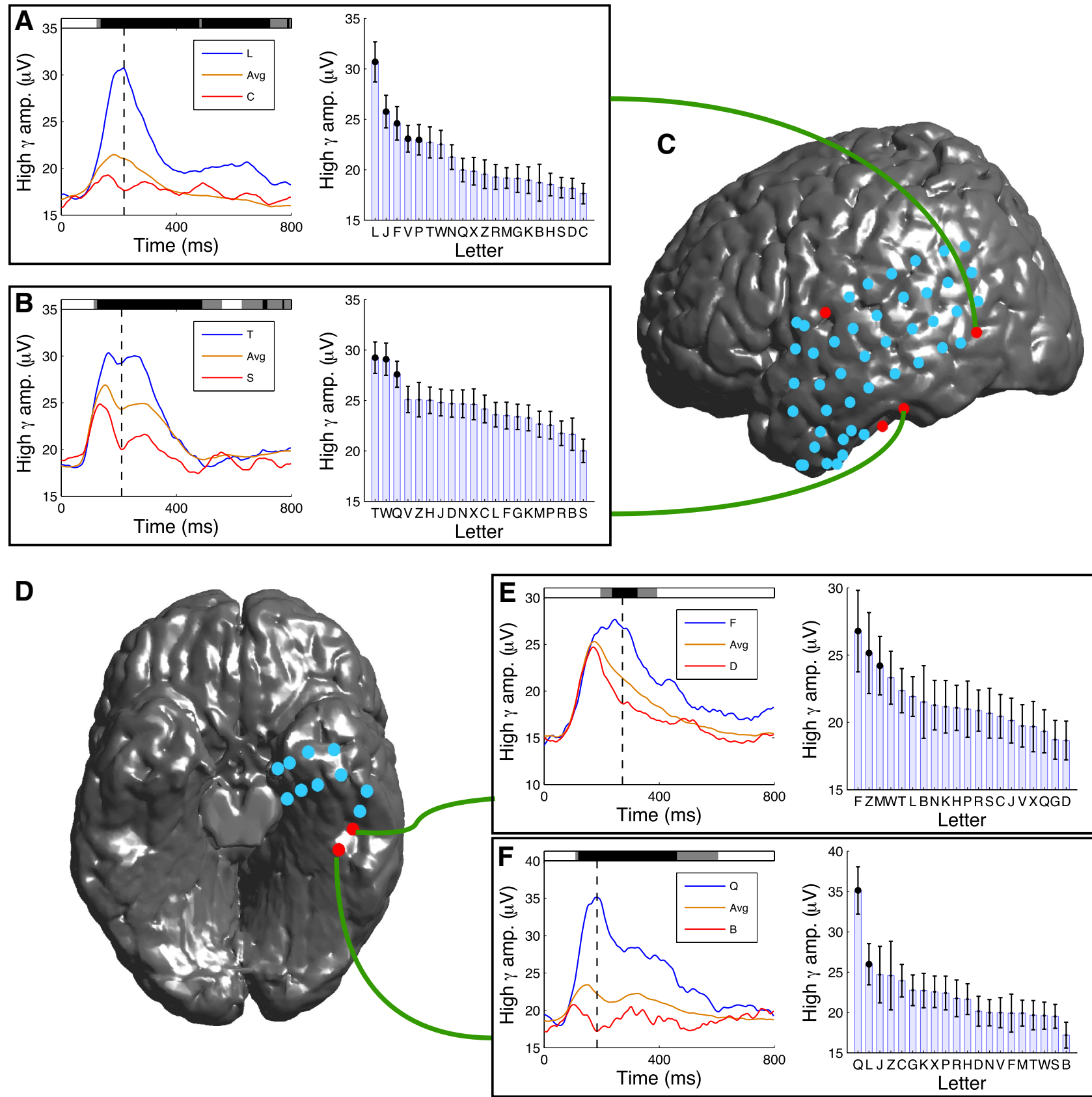

Figure 4. Examples of letter-related high-gamma $(65-128 \mathrm{~Hz})$ activity. $A$, A letter-sensitive electrode from the left temporal cortex of patient 20 (Brodmann area 21$)$ that exhibited the largest high-gamma amplitude after viewing of the letter L (same electrode as Fig. 3). Left depicts the time course of the mean high-gamma amplitude for letters $\mathrm{L}$ (blue) and ( (red) and the mean amplitude for all letters (orange). Black dashed line indicates the time point in which this effect is largest. Shaded regions at top of plot indicate the time points of significant clusters of letter-related activity (gray coloring indicates letter-related differences at $p<0.05$; black indicates $p<10^{-5}$ ). Right indicates the mean high-gamma power for each letter at the time point of peak letter-related differences (indicated by the black dashed line in the left). Error bars denote $95 \%$ confidence intervals. Black dots indicate that the gamma amplitude of a letter was significantly elevated compared with all other letters at that time point ( post hoc one-sided $t$ tests, $p$ values $<0.05$ ). $\boldsymbol{B}$, A letter-sensitive electrode from patient 20's left temporal cortex (Brodmann area 36) that had the largest high-gamma activity for letter T. C, Placement of electrodes on patient 20's left neocortex. Red dots indicate electrodes that exhibited significant letter-related gamma activity, and teal dots indicate electrodes that did not exhibit significant letter-related gamma activity. Curved green lines connect panels to the corresponding electrode. $\boldsymbol{D}$, Inferior view of patient 4 's electrodes. $\boldsymbol{E}$, The activity of a letter-sensitive electrode from patient 4's left-temporal cortex (Brodmann area 37) that exhibited the largest-amplitude high-gamma oscillations after viewing the letter F. $F$, An electrode from patient 4's left occipital cortex (Brodmann area 19) that exhibited largest high-gamma amplitude when viewing letter Q.

cant theta phase locking. This phase synchrony could result from a phase reset in an ongoing theta oscillation (Rizzuto et al., 2003) or from a new evoked theta waveform (Fell et al., 2004). At these sites, we observed an increased prevalence of letter-related gamma activity and gamma-amplitude increases $(\Phi=0.11$; $\left.\chi_{(1)}^{2}=30 ; p<10^{-7}\right)$. Furthermore, we found that, across electrodes exhibiting both effects, the time point of peak theta-phase synchrony was closely related to the onset time of elevated gamma amplitude (Kendall's $\tau=0.24 ; p<10^{-6}$ ). Because different neural processes occur at distinct theta phases (Chrobak and Buzsáki, 1998; Canolty et al., 2006; Tort et al., 2008), we examined the relation between theta phase and the emergence of letter-related gamma activity. For each electrode exhibiting both theta phase reset and letter-related gamma activity, we 

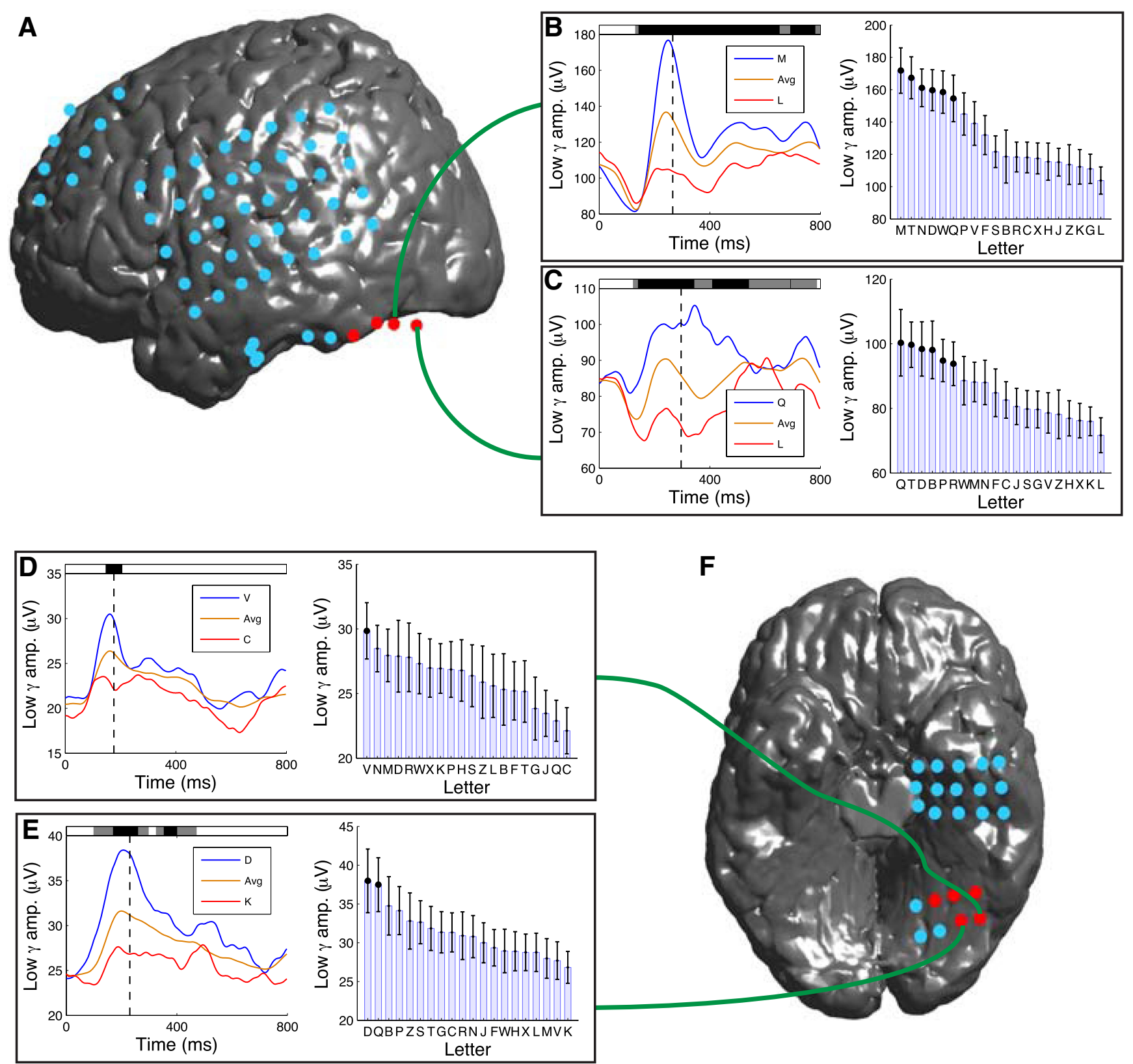

Figure 5. Examples of letter-related low-gamma (25-55 Hz) activity. A, Electrode locations on patient 24 's left neocortex. $B$, A letter-sensitive electrode from the left occipital cortex of patient 24 (Brodmann area 19) that exhibited the largest low-gamma oscillations after viewing of the letter M. C, The activity of a letter-sensitive electrode from patient 24 's left occipital cortex that exhibited the highest-amplitude low-gamma activity after viewing the letter Q. D. A letter-sensitive electrode from patient 21's left occipital cortex (Brodmann area 19) that exhibited the highest amplitude low-gamma activity after viewing of the letter V. E, A letter-sensitive electrode from patient 21's left occipital cortex (Brodmann area 19) that exhibited the highest-amplitude low-gamma activity after viewing of the letter D. $\boldsymbol{F}$, Inferior view of patient 21 's electrodes.

separately calculated the mean theta phase at the time of the overall gamma power increase (Fig. 6A) and at the time of the emergence of letter-related activity (Fig. $6 B$ ). As a result, we found that the initial gamma-amplitude increases occurred just before the peak phase of theta (mean phase $\mu=5.9 \mathrm{rad}$; $p<0.002$, Rayleigh test) and that the emergence of letterspecific gamma activity occurred on the descending phase of the theta cycle $(\mu=0.9 \mathrm{rad} ; p<0.02)$. We also conducted a general analysis of the relation between theta phase and gamma amplitude (Canolty et al., 2006), indicating that overall gamma amplitude in our dataset was maximal at the trough phase of theta (Fig. 6C). Together, these findings suggest that, at sites exhibiting letter-specific gamma activity, gamma am- plitude first significantly increases at the peak phase of theta and then this increase continues until the theta trough.

Next we examined whether letter-specific gamma oscillations were prevalent in particular brain regions (Fig. 7). We found that the prevalence of letter-related gamma activity significantly varied across the brain $\left(\chi_{(4)}^{2}=136 ; p<10^{-15}\right)$. Specifically, we found that the number of electrodes exhibiting letter-specific gamma activity significantly exceeded the level expected by chance in occipital and temporal cortices ( $p$ values $<0.05$, using a permutation test corrected for multiple comparisons). In occipital cortex, a significantly greater proportion of electrodes exhibited letter-specific activity than in temporal cortex $(p<0.001$, permutation test). Although a slightly greater pro- 
A

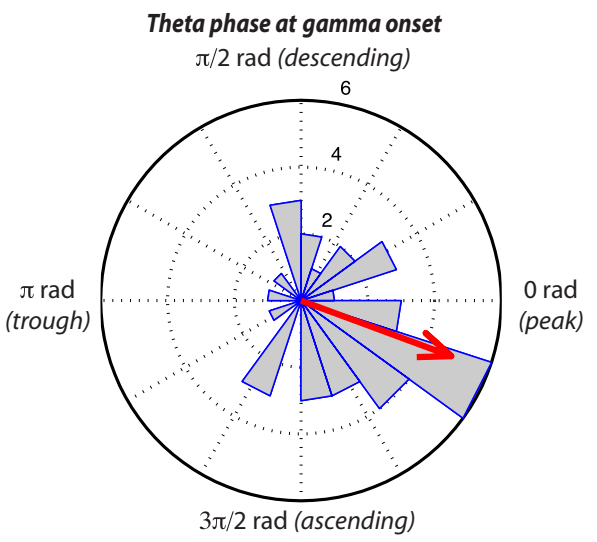

B

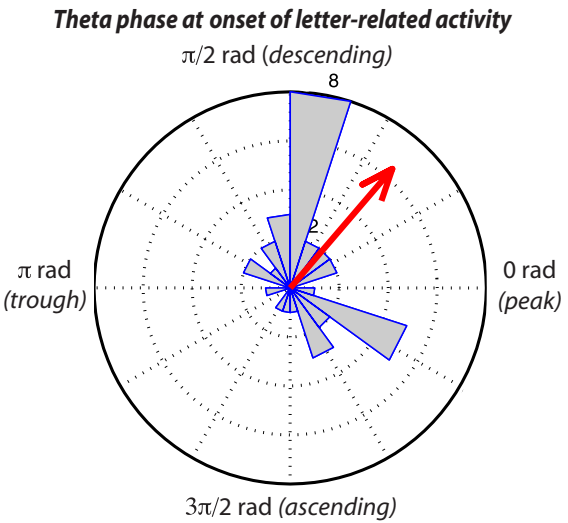

C

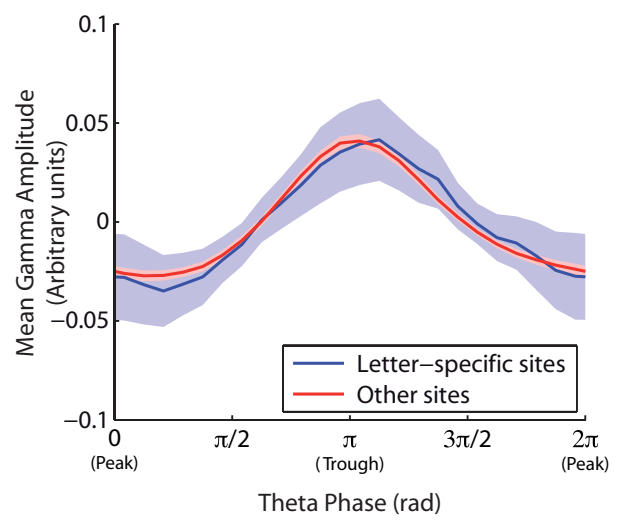

Figure 6. The relation between letter-related gamma activity and theta phase, computed across all electrodes that exhibited a letter-related gamma-power increase and theta phase locking. $A$, Circular histogram indicating the distribution of mean theta phases corresponding to the onset times of elevated gamma activity at individual electrodes. Red arrow indicates the overall mean phase. $\boldsymbol{B}$, The distribution of mean theta phases corresponding to the onset times of letter-specific gamma activity. $\boldsymbol{C}$, Mean high- and low-gamma amplitude as a function of instantaneous theta phase, computed for electrodes exhibiting significant letter-specific activity (blue) and for other sites (red). Shaded regions indicate 95\% confidence intervals.

portion of the observed letter-specific activity appeared in the low-gamma band, in both occipital (56\%) and temporal (60\%) cortices, we could not reject the hypotheses that letter-specific activity in these regions was equally prevalent in the low- and high-gamma bands (binomial tests, $p$ values $>0.3$ ). Note that, although the occipital cortex had the largest proportion of electrodes exhibiting letter-specific activity, our dataset contained a relatively small number of electrodes in this region. Because of this uneven sampling, the counts of electrodes that we observed exhibiting letter-specific activity in occipital and temporal corti- ces were comparable (supplemental table, available at www. jneurosci.org as supplemental material).

In individual patients, we often observed that letter-specific activity appeared at neighboring groups of electrodes (Figs. 4, 5). To examine this phenomenon quantitatively, we used Moran's $I$ (Moran, 1950), a measure of spatial autocorrelation across observations made at different locations (for review, see Ripley, 1981). Across the entire dataset, we found that sites exhibiting letterspecific activity were not uniformly distributed and instead occurred in spatially proximal groups $\left(I=0.07 ; p<10^{-4}\right)$. This clustering was also present when we separately analyzed the electrodes from occipital $(I=0.16 ; p=0.005)$ and temporal $(I=0.05 ; p<0.005)$ cortices. Next, beyond examining spatial clustering of the sites that exhibited letter-specific activity, we examined whether there were additional spatial correlations among the properties of these sites. Including only the electrodes exhibiting letter-specific activity, there was a significant spatial autocorrelation in the frequency (high or low gamma) at which this pattern occurred $\left(I=0.11 ; p<10^{-4}\right)$. This indicates that, when neighboring electrodes exhibited letter-specific activity, they often demonstrated this effect at the same frequency band. Furthermore, there was a significant spatial correlation between the time points of peak letter specificity observed at nearby electrodes $(I=0.22 ; p=0.01)$. Thus, when neighboring electrodes exhibited letter-specific activity, these patterns often occurred at similar time points.

Having characterized the spatial and temporal properties of letter-specific gamma activity, we next aimed to identify the nature of the information coded by these patterns. Does this gamma activity reflect low-level perceptual information about the shape of the viewed letter or does it reflect more-abstract cognitive representations? To examine this issue, we analyzed the relation between the gamma activity pattern for each letter and the perceptual properties of the letter (i.e., the shape of a letter). We used data from a behavioral study of visual perception (S. T. Mueller and C. T. Weidemann, unpublished observation), which measured the perceptual dissimilarity between letter pairs. Letters with similar shapes (e.g., $\mathrm{M}$ and $\mathrm{N}$ ) were judged as being a short perceptual distance from each other, and dissimilar letters (e.g., $\mathrm{X}$ and $\mathrm{O}$ ) were judged as being perceptually distant. We also computed the absolute difference between the mean gamma amplitudes for each letter pair (the "gamma distance"). To determine whether the letter-related gamma responses of an electrode encoded information about the shape of the just-presented letter, we compared the perceptual and gamma distances of each letter pair. If these two sets of distances were significantly positively correlated, it indicates that the gamma activity of the electrode reflected the shape of the letter.

This analysis identified several letter-sensitive electrodes that encoded information about the shape of the viewed letter. As an example, Figure $8 \mathrm{~A}$ depicts the activity of a right-occipital electrode that exhibited peak letter-related high-gamma activity 168 $\mathrm{ms}$ after stimulus presentation. At that time point, the pattern of letter-related gamma responses positively correlated with the perceptual representations of the letters $\left(r=0.45 ; p<10^{-3}\right)$. Specifically, this electrode had significantly elevated gamma amplitude for round letters C, D, and $\mathrm{G}$ and had less gamma activity for letters with straight lines (e.g., $\mathrm{N}$ and $\mathrm{K}$ ). To identify regions in which many electrodes exhibited perception-related gamma activity, we computed the proportion of electrodes in each brain lobe that significantly demonstrated this effect ( $p$ values $<0.01$ ). The prevalence of this phenomenon varied significantly throughout the brain $\left(\chi_{(4)}^{2}=13 ; p<0.01\right)$, and the only 
A

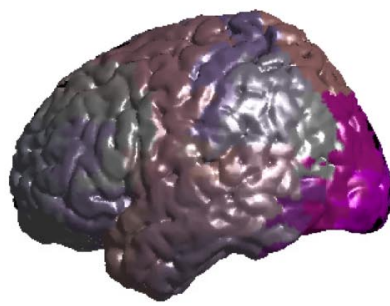

B

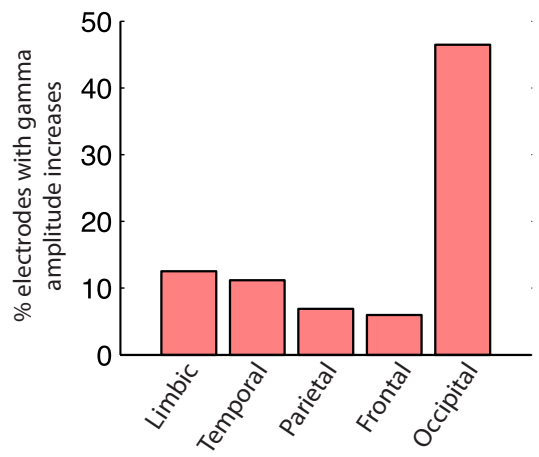

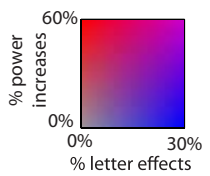

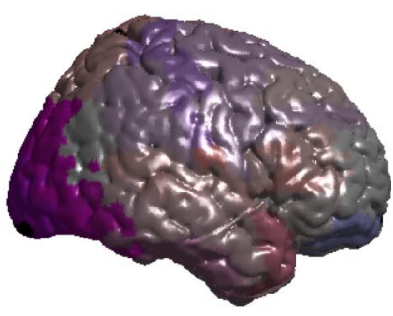

C

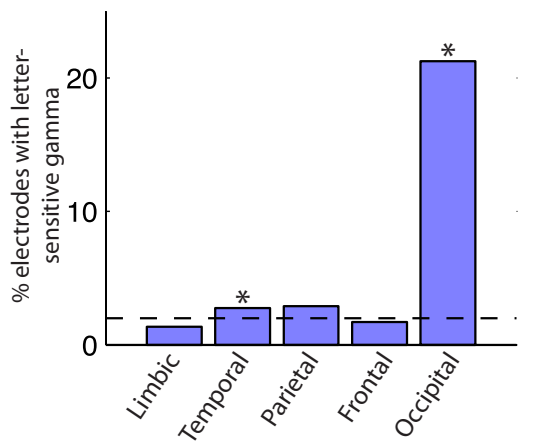

Figure 7. Regional distribution of electrodes exhibiting letter-related gamma activity. $\boldsymbol{A}$, Left and right views of the neocortex indicating the percentages of electrodes in each region exhibiting letter-related high- or low-gamma activity. Percentages are plotted with a bivariate color scheme in which red coloring indicates the percentage of electrodes exhibiting overall increases in gamma amplitude, and blue coloring indicates the percentage of electrodes exhibiting letter-related activity (purple indicates that both effects were present). $\boldsymbol{B}$, The percentage of electrodes in each brain region exhibiting overall increases in gamma amplitude. $\boldsymbol{C}$, The percentage of electrodes in each region exhibiting letter-related gamma activity. Asterisks indicate regions in which the proportion of observed letter-responsive electrodes significantly exceeded the level expected by chance (dashed black line) at $p<$ 0.05 according to a permutation procedure corrected for multiple comparisons.

regions in which significant numbers of electrodes exhibited this pattern were occipital and parietal cortices (one-sided binomial tests, $p$ values $<0.05$ ). In occipital cortex, of the 27 electrodes that exhibited letter-related gamma activity, five (19\%) significantly encoded information about letter shape.

\section{Discussion}

We conducted an item-level analysis of oscillatory activity in human ECoG recordings and observed 78 electrodes in which gamma-band activity significantly encoded the identity of the currently viewed letter. This shows that human gamma ECoG activity not only identifies general brain regions that are actively engaged in a particular behavior, but it also reveals temporal and spatial details of the cortical representations for individual stimuli. Below, we discuss the relation between these patterns and the existing literature on the electrophysiology of human cognition, and we comment on the implications of these findings for future research.

In recent years, a growing literature has examined human ECoG recordings to learn about the neuronal activity that underlies cognition. One success of this research is the identification of oscillatory patterns, at various frequency bands including gamma, that distinguish broad classes of human cognitive processes, such as memory formation, decision making, and processing of different types of stimuli (Buzsáki, 2006; Kahana, 2006). These patterns identified the brain regions that support various neural computations (Crone et al., 1998; Bauer et al., 2006; Miller et al., 2007; Ray et al., 2007) and even revealed neuronal communication across regions (Engel et al., 1991; Canolty et al., 2007). In a different set of research, studies of animal perception showed that gamma oscillations revealed specific attributes of distinct percepts (Freeman, 1991; Singer and Gray, 1995), which suggested to us that recordings of gamma activity may reveal how the human brain encodes details of individual stimuli.

Our findings support the view that gamma-band activity is involved in various neural patterns in addition to lowlevel perception. Although we observed some electrodes in which gamma activity appeared to encode the visual appearances of letters, other letter-sensitive electrodes responded to different information besides letter shape. A related set of findings comes from item-level function magnetic resonance imaging (fMRI) studies showing that neuronal activity in visual cortex encodes perceptual properties of visual stimuli (Kamitani and Tong, 2005), whereas activity in other regions encodes more-abstract stimulus features (Mitchell et al., 2008). To the extent that fMRI activations are correlated with gamma activity (Logothetis et al., 2001), these findings are consistent with our results.

An important part of our findings is the discovery that letter-specific patterns were essentially limited to the regions showing overall increases in gamma activity. These overall gamma increases appear to be indications of general stimulusrelated increases in cortical network processing (Freeman, 1975). Our results suggest that letter-related gamma activity only potentially emerges after these networks have been engaged for $\sim 50-100 \mathrm{~ms}$. Thus, a useful strategy for identifying stimulus-related activity in future electrophysiological studies is first to identify the regions and time points that exhibit overall stimulus-related gammaamplitude increases. Then, in subsequent data analyses, the activity in these regions should be analyzed at a slightly later latency. Because gamma-band activity is visible with scalp electroencephalography and magnetoencephalography and is correlated with the signals observed in fMRI and near-infrared-spectroscopy studies (Logothetis et al., 2001; Moosmann et al., 2003), this twostep screening procedure may be useful in various types of brain research.

Our findings suggest that multiple brain regions are involved in representing stimulus-specific information. The region in which the greatest proportion of electrodes exhibited letterspecific activity was occipital cortex. This might be expected given the visual nature of our task and the established retinotopic mapping of cortical columns in this region (Holmes, 1918). Indeed, a different presentation modality (e.g., auditory or tactile presentation) might have led to maximal levels of letter-related activity in a different region (e.g., auditory or somatosensory cortex). We also observed significant letter-specific activity in temporal cortex, suggesting that detailed information about visually presented stimuli is encoded in widespread brain networks that may encode both shape-specific and more-abstract information. Consistent with this, other recent studies also found memory-related neuronal activity in regions not typically associated with memory, such as motor cortex (Heekeren et al., 2008). 
Thus, gamma-band activity may be useful for precisely mapping differences in the neural representations of cognitive information across individuals.

The letter-specific gamma patterns we observed are reminiscent of the "wave packets" described by Freeman (2003). In animals, wave packets are high-amplitude gamma oscillations that are phase locked across relatively large areas $\left(\geq 1 \mathrm{~cm}^{2}\right)$ of cortex. The oscillations are a distributed pattern that indicates the current state of the local cortical neuronal network via differences in gamma amplitude across sites. Wave packets have been shown to play a critical role in associative learning because they encode the learned meaning of percepts (Ohl et al., 2001). Although they have been observed in many animal species, the evidence for wave packets in humans is unclear (Menon et al., 1996).

In contrast to the wave packets described by Freeman, which encode information in a distributed, multivariate manner, here we exclusively conducted univariate analyses of gamma activity at individual sites. Nonetheless, both findings seem closely related because they each describe stimulus-specific patterns of gamma-amplitude modulation that occur during periods of elevated overall gamma activity and that are linked to theta oscillations (Freeman et al., 2003). We sometimes observed that neighboring electrodes exhibited letter-specific activity at similar time points but that each site varied in its response to individual letters.

This would be expected from a single wave packet spanning these sites. Menon et al. (1996) searched for wave packets in ECoG recordings from two patients, but the findings were inconclusive and the authors concluded that the standard $\sim 1 \mathrm{~cm}$ spacing of standard neurosurgical ECoG electrodes was too coarse to identify wave packets. Although our recording configuration was similar, our findings are qualitatively different. One potential cause of this difference is that the letter stimuli we used were more familiar and meaningful to patients than the relatively novel somatosensory stimuli used previously.

Recent research suggests that the interplay between theta and gamma activity plays an important role in human cognition (Lisman and Idiart, 1995; Freeman et al., 2003; Mormann et al., 2005; Canolty et al., 2006; Sirota et al., 2008). Generally, gamma activity has the largest amplitude at the trough of theta (Canolty et al., 2006), a finding that we replicated (Fig. 6C). In addition, we found that the latency of theta phase locking correlated with the onset time of increased gamma activity (Başar et al., 1987), which is also consistent with the notion that theta and gamma activity are tightly coupled. We observed that, at sites exhibiting letter-related activity, gamma amplitude first exhibits significant elevation at the peak phase of theta rather than at the trough. Furthermore, Mormann et al. (2005) (Fig. 5) also show stimulus-related gamma oscillations beginning at the peak phase of theta. Together, these findings suggest that processing a particular stimulus can cause significant gamma activity that be-
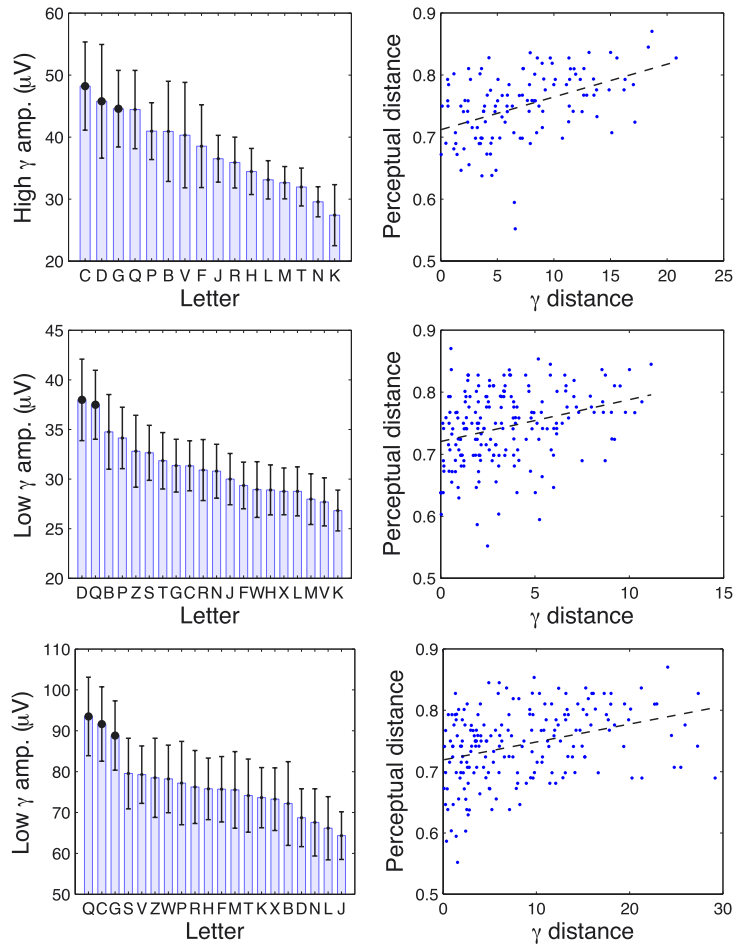

Figure 8. Relation between perceptual similarity and letter-specific gamma-band responses. $\boldsymbol{A}$, Example electrode from patient 25 's right occipital cortex. Left depicts the time course of high-gamma activity and the time points exhibiting significant ces for each pair of letters. Each dot corresponds to a pair of two letters. The vertical position of each dot indicates the discriminability. C, An electrode from patient 18's right occipital cortex that exhibits a significant positive correlation between low-gamma activity and perceptual discriminability.

gins at the peak phase of theta. After this initial increase, the amplitude of this activity continues to increase until the theta trough. In this way, although there is significant gamma activity at various theta phases, the largest gamma amplitude nonetheless occurs at the theta trough (Canolty et al., 2006).

Here we analyzed oscillatory ECoG activity in two separate frequency bands: the 25-55 Hz low-gamma band and the 65-128 $\mathrm{Hz}$ high-gamma band. We chose these bands because of our initial analyses (Fig. 1D) and several recent studies (Rickert et al., 2005; Vidal et al., 2006; Sederberg et al., 2007; Wyart and TallonBaudry, 2008), which showed that neuronal activity in each of these bands often have different functional roles. Recently, Miller et al. (2007) suggested that some apparent high-gamma EcoG activity is actually caused by broadband spectral shifts resulting from overall increases in synaptic activity rather than by bona fide oscillations. Thus, it is possible that some of our results are driven by stimulus-related increases in local neuronal activity, which are especially visible in the high-gamma band rather than by true oscillations (Ray et al., 2008; J. Manning, J. Jacobs, I. Fried, and M. Kahana, unpublished observation). In practice, it can be difficult to distinguish gamma oscillations from broadband spectral shifts, because both phenomena are correlated with increased cortical activity and both patterns often appear in wide, overlapping frequency ranges (Siegel and König, 2003; Rickert et al., 2005; Vidal et al., 2006; Miller et al., 2007; Kaiser et al., 2008; Chandrasekaran and Ghazanfar, 2009). Thus, an important task 
for future research is to carefully distinguish the physiological and functional properties of each of these phenomena. More broadly, recent human brain research shows that the range of frequencies that encodes behavioral information can vary across patients, brain regions, tasks, and even different parts of a task (Klimesch, 1999; Vidal et al., 2006; Miller et al., 2009). To identify these patterns more precisely, future studies may need to systematically map the particular frequency range that encodes task-related information at each recording site (Siegel and König, 2003).

Although our current findings are limited to data from a task in which patients viewed letters, similar patterns might appear for other stimulus types, such as pictures, cartoons, or words. In some patients, we found letter-specific activity at several neighboring electrodes spanning many square centimeters of cortex; we do not believe that these entire regions are uniquely specialized for representing letters. Rather, we believe that our findings are indications of large, diverse cortical networks that may represent various classes of stimuli. There is clear evidence that various regions of cortex specifically activate when processing different types of stimuli, such as faces (Kanwisher et al., 1997) or even letters (Polk et al., 2002), in addition to more-general regions that respond to various stimulus classes (Mitchell et al., 2008). Our findings suggest that gamma-band activity can reveal the nature of neuronal computations across a wide range of brain regions.

\section{References}

Başar E, Rosen B, Başar-Eroglu C, Greitschus F (1987) The associations between $40 \mathrm{~Hz}$-EEG and the middle latency response of the auditory evoked potential. Int J Neurosci 33:103-117.

Bauer M, Oostenveld R, Peeters M, Fries P (2006) Tactile spatial attention enhances gamma band activity in somatosensory cortex and reduces lowfrequency activity in parieto-occipital areas. J Neurosci 26:490-501.

Bressler SL, Freeman WJ (1980) Frequency analysis of olfactory system EEG in cat, rabbit, and rat. Electroencephalogr Clin Neurophysiol 50:19-24.

Buzsáki G (2006) Rhythms of the brain, New York: Oxford UP.

Canolty RT, Edwards E, Dalal SS, Soltani M, Nagarajan SS, Kirsch HE, Berger MS, Barbaro NM, Knight RT (2006) High gamma power is phaselocked to theta oscillations in human neocortex. Science 313:1626-1628.

Canolty RT, Soltani M, Dalal SS, Edwards E, Dronkers NF, Nagarajan SS, Kirsch HE, Barbaro NM, Knight RT (2007) Spatiotemporal dynamics of word processing in the human brain. Front Neurosci 1:185-196.

Chandrasekaran C, Ghazanfar AA (2009) Different neural frequency bands integrate faces and voices differently in the superior temporal sulcus. J Neurophysiol 101:773-788.

Chrobak JJ, Buzsáki G (1998) Gamma oscillations in the entorhinal cortex of the freely behaving rat. J Neurosci 18:388-398.

Crone NE, Miglioretti DL, Gordon B, Sieracki JM, Wilson MT, Uematsu S, Lesser RP (1998) Functional mapping of human sensorimotor cortex with electrocorticographic spectral analysis. I. Alpha and beta eventrelated desynchronization. Brain 121:2271-2299.

Eckhorn R, Bauer R, Jordan W, Brosch M, Kruse W, Munk M, Reitboeck HJ (1988) Coherent oscillations: a mechanism of feature linking in the visual cortex? Biol Cybern 60:121-130.

Engel A, Konig P, Kreiter A, Singer W (1991) Interhemispheric synchronization of oscillatory neuronal responses in cat visual cortex. Science 252:1177-1179.

Fell J, Dietl T, Grunwald T, Kurthen M, Klaver P, Trautner P, Schaller C, Elger CE, Fernández G (2004) Neural bases of cognitive ERPs: more than phase reset. J Cogn Neurosci 16:1595-1604.

Freeman W (1975) Mass action in the nervous system. San Diego: Academic.

Freeman W (2007) Hilbert transform for brain waves. Scholarpedia 2:1338.

Freeman WJ (1991) The physiology of perception. Sci Am 264:78-85.

Freeman WJ (2003) The wave packet: an action potential for the 21 st century. J Integr Neurosci 2:3-30.

Freeman WJ, Burke BC, Holmes MD (2003) A periodic phase re-setting in scalp EEG of beta-gamma oscillations by state transitions at alpha-theta rates. Hum Brain Mapp 19:248-272.

Galton F (1883) Inquiries into human faculty and its development. London: Macmillan and Co.
Genovese CR, Lazar NA, Nichols TE (2002) Thresholding of statistical maps in functional neuroimaging using the false discovery rate. Neuroimage 15:870-878.

Graboi D, Lisman J (2003) Recognition by top-down and bottom-up processing in cortex: the control of selective attention. J Neurophysiol 90:798-810.

Gray CM, König P, Engel AK, Singer W (1989) Oscillatory responses in cat visual cortex exhibit inter-columnar synchronization which reflects global stimulus properties. Nature 338:334-337.

Hebb DO (1949) Organization of behavior. New York: Wiley.

Heekeren HR, Marrett S, Ungerleider LG (2008) The neural systems that mediate human perceptual decision making. Nat Rev Neurosci 9:467-479.

Holmes G (1918) Disturbances of vision by cerebral lesions. Br Med J 2:353. Howard MW, Rizzuto DS, Caplan JB, Madsen JR, Lisman J, AschenbrennerScheibe R, Schulze-Bonhage A, Kahana MJ (2003) Gamma oscillations correlate with working memory load in humans. Cereb Cortex 13:1369-1374.

Jacobs J, Kahana MJ, Ekstrom AD, Fried I (2007) Brain oscillations control timing of single-neuron activity in humans. J Neurosci 27:3839-3844.

Jacobs J, Korolev I, Caplan J, Ekstrom A, Litt B, Baltuch G, Fried I, SchulzeBonhage A, Madsen J, Kahana M (2009) Right-lateralized brain oscillations in human spatial navigation. J Cogn Neurosci. Advance online publication. Retrieved June 17, 2009. doi:10.1162/jocn.2009.21240.

Kahana MJ (2006) The cognitive correlates of human brain oscillations. J Neurosci 26:1669-1672.

Kaiser J, Heidegger T, Wibral M, Altmann CF, Lutzenberger W (2008) Distinct gamma-band components reflect the short-term memory maintenance of different sound lateralization angles. Cereb Cortex 18:2286-2295.

Kamitani Y, Tong F (2005) Decoding the visual and subjective contents of the human brain. Nat Neurosci 8:679-685.

Kanwisher N, McDermott J, Chun MM (1997) The fusiform face area: a module in human extrastriate cortex specialized for face perception. J Neurosci 17:4302-4311.

Klimesch W (1999) EEG alpha and theta oscillations reflect cognitive and memory performance: a review and analysis. Brain Res Rev 29:169-195.

Lancaster JL, Woldorff MG, Parsons LM, Liotti M, Freitas CS, Rainey L, Kochunov PV, Nickerson D, Mikiten SA, Fox PT (2000) Automated Talairach atlas labels for functional brain mapping. Hum Brain Mapp $10: 120-131$.

Lashley K (1950) In search of the engram. Symp Soc Exp Biol 4:30.

Lisman JE, Idiart MA (1995) Storage of $7 \pm 2$ short-term memories in oscillatory subcycles. Science 267:1512-1515.

Liu H, Agam Y, Madsen JR, Kreiman G (2009) Timing, timing, timing: fast decoding of object information from intracranial field potentials in human visual cortex. Neuron 62:281-290.

Llinás R, Ribary U, Contreras D, Pedroarena C (1998) The neuronal basis for consciousness. Philos Trans R Soc Lond B Biol Sci 353:1841-1849.

Logothetis NK, Pauls J, Augath M, Trinath T, Oeltermann A (2001) Neurophysiological investigation of the basis of the fMRI signal. Nature 412:150-157.

Maldjian JA, Laurienti PJ, Kraft RA, Burdette JH (2003) An automated method for neuroanatomic and cytoarchitectonic atlas-based interrogation of fMRI data sets. Neuroimage 19:1233-1239.

Maris E, Oostenveld R (2007) Nonparametric statistical testing of EEG- and MEG-data. J Neurosci Methods 164:177-190.

Melton A, Martin E (1972) Coding processes in human memory. New York: Halsted.

Menon V, Freeman WJ, Cutillo BA, Desmond JE, Ward MF, Bressler SL, Laxer KD, Barbaro N, Gevins AS (1996) Spatio-temporal correlations in human gamma band electrocorticograms. Electroencephalogr Clin Neurophysiol 98:89-102.

Miller KJ, Leuthardt EC, Schalk G, Rao RP, Anderson NR, Moran DW, Miller JW, Ojemann JG (2007) Spectral changes in cortical surface potentials during motor movement. J Neurosci 27:2424-2432.

Miller KJ, Zanos S, Fetz EE, den Nijs M, Ojemann JG (2009) Decoupling the cortical power spectrum reveals real-time representation of individual finger movements in humans. J Neurosci 29:3132-3137.

Mitchell TM, Shinkareva SV, Carlson A, Chang KM, Malave VL, Mason RA, Just MA (2008) Predicting human brain activity associated with the meanings of nouns. Science 320:1191-1195.

Moosmann M, Ritter P, Krastel I, Brink A, Thees S, Blankenburg F, Taskin B, 
Obrig H, Villringer A (2003) Correlates of alpha rhythm in functional magnetic resonance imaging and near infrared spectroscopy. Neuroimage 20:145-158.

Moran P (1950) Notes on continuous stochastic phenomena. Biometrika 37:17-33.

Mormann F, Fell J, Axmacher N, Weber B, Lehnertz K, Elger CE, Fernández G (2005) Phase/amplitude reset and theta-gamma interaction in the human medial temporal lobe during a continuous word recognition memory task. Hippocampus 15:890-900.

Ohl FW, Scheich H, Freeman WJ (2001) Change in pattern of ongoing cortical activity with auditory category learning. Nature 412:733-736.

Polk TA, Stallcup M, Aguirre GK, Alsop DC, D’Esposito M, Detre JA, Farah MJ (2002) Neural specialization for letter recognition. J Cogn Neurosci 14:145-159.

Raghavachari S, Kahana MJ, Rizzuto DS, Caplan JB, Kirschen MP, Bourgeois B, Madsen JR, Lisman JE (2001) Gating of human theta oscillations by a working memory task. J Neurosci 21:3175-3183.

Ray S, Niebur E, Hsiao SS, Sinai A, Crone NE (2007) High-frequency gamma activity $(80-150 \mathrm{~Hz})$ is increased in human cortex during selective attention. Clin Neurophysiol 119:116-133.

Ray S, Crone NE, Niebur E, Franaszczuk PJ, Hsiao SS (2008) Neural correlates of high-gamma oscillations $(60-200 \mathrm{~Hz})$ in macaque local field potentials and their potential implications in electrocorticography. J Neurosci 28:11526-11536.

Rickert J, Oliveira SC, Vaadia E, Aertsen A, Rotter S, Mehring C (2005) Encoding of movement direction in different frequency ranges of motor cortical local field potentials. J Neurosci 25:8815-8824.

Ripley B (1981) Spatial statistics. New York: Wiley-Interscience.

Rizzuto DS, Madsen JR, Bromfield EB, Schulze-Bonhage A, Seelig D, Aschenbrenner-Scheibe R, Kahana MJ (2003) Reset of human neocortical oscillations during a working memory task. Proc Natl Acad Sci U S A 100:7931-7936.

Sederberg PB, Kahana MJ, Howard MW, Donner EJ, Madsen JR (2003) Theta and gamma oscillations during encoding predict subsequent recall. J Neurosci 23:10809-10814.
Sederberg PB, Schulze-Bonhage A, Madsen JR, Bromfield EB, McCarthy DC, Brandt A, Tully MS, Kahana MJ (2007) Hippocampal and neocortical gamma oscillations predict memory formation in humans. Cereb Cortex 17:1190-1196

Siegel M, König P (2003) A functional gamma-band defined by stimulusdependent synchronization in area 18 of awake behaving cats. J Neurosci 23:4251-4260.

Singer W, Gray CM (1995) Visual feature integration and the temporal correlation hypothesis. Annu Rev Neurosci 18:555-586.

Sirota A, Montgomery S, Fujisawa S, Isomura Y, Zugaro M, Buzsáki G (2008) Entrainment of neo-cortical neurons and gamma oscillations by the hippocampal theta rhythm. Neuron 60:683-697.

Sternberg S (1966) High-speed scanning in human memory. Science 153:652-654

Talairach J, Tournoux P (1988) Co-planar stereotaxic atlas of the human brain. Stuttgart, Germany: Thieme.

Tononi G, Koch C (2008) The neural correlates of consciousness: an update. Ann N Y Acad Sci 1124:239-261.

Tort AB, Kramer MA, Thorn C, Gibson DJ, Kubota Y, Graybiel AM, Kopell NJ (2008) Dynamic crossfrequency couplings of local field potential oscillations in rat striatum and hippocampus during performance of a T-maze task. Proc Natl Acad Sci U S A 105:20517-20522.

Tovée MJ, Rolls ET, Treves A, Bellis RP (1993) Information encoding and the responses of single neurons in the primate temporal visual cortex. J Neurophysiol 70:640-654.

van Vugt MK, Schulze-Bonhage A, Sekuler R, Litt B, Brandt A, Baltuch G, Kahana MJ (2009) Intracranial electroencephalography reveals two distinct two similarity effects during item recognition. Brain Res. Retrieved June 17, 2009. doi:10.1016/j.brainres.2009.07.016.

Vidal JR, Chaumon M, O'Regan JK, Tallon-Baudry C (2006) Visual grouping and the focusing of attention induce gamma-band oscillations at different frequencies in human magnetoencephalogram signals. J Cogn Neurosci 18:1850-1862.

Wyart V, Tallon-Baudry C (2008) Neural dissociation between visual awareness and spatial attention. J Neurosci 28:2667-2679. 